\title{
Conclusion La dimension collective de l'élection : des impensés du don électoral au vécu de la campagne en groupe
}

Guillaume Courty et Julie Gervais L'éruption du Vésuve à Pompéi a figé sur les murs de la ville ce que la pluie, les touristes et d'autres déprédations ont effacé dans les autres sites urbains romains : des messages concernant les candidats ${ }^{1}$ en lice, peints à l'occasion des élections. Ces dipinti montrent à quel point une campagne électorale était « vivante » et offrait à « des personnes seules et des familles, des associations professionnelles et des voisins ${ }^{2}$ » l'occasion de s'exprimer sur les candidats et d'en soutenir certains. K.-W. Weeber se livre à leur analyse, soulignant que de telles inscriptions ne manqueraient pas de susciter un « tollé ${ }^{3}$ » si de nombreux acteurs collectifs s'y adonnaient à une grande échelle lors des élections contemporaines. Cet exemple d'enquête électorale éloigné du cas français contemporain vient signaler qu'il y a beaucoup à puiser dans les recherches menées ailleurs en sciences sociales pour analyser ici la participation des groupes au déroulé des campagnes électorales. Plusieurs propriétés distinctives (telles que la morphologie sociale, les institutions gouvernementales et fédérales, la structure des partis, etc.) militent pourtant contre l'emprunt de certains cadres d'analyse ou l'analogie avec certaines pièces du répertoire d'actions électorales. C'est donc « toutes choses égales par ailleurs » que nous proposons un dernier retour sur les terres électorales étatsuniennes, en y ajoutant un ultime plaidoyer en faveur, cette fois, de l'ouverture à l'anthropologie.

Ce retour nous paraît nécessaire afin d'explorer une dernière question fondamentale : qu'attendent les groupes en campagne en retour de leur action électorale ? Les enquêtes indiquent que le financement des candidats et des partis par les groupes d'intérêt s'inscrit dans leur quête d'influence. L'analyse consiste alors à rechercher si, et dans quelle mesure, le financement est lié à l'élaboration d'un thème de campagne, à la

1 Nous avons choisi ici de ne « genrer » que les termes au singulier.

2Weeber K.-W., Fièvre électorale à Pompéi, Paris, Les Belles Lettres, 2011 [1ère éd. 2007], p. 8.

3Ibid., p. 35. 
carrière d'un enjeu ou aux positions tenues pendant la session parlementaire. Cette réponse, nous l'avons dit, nous semble insatisfaisante (cf. chapitre 1). Elle est d'abord révélatrice du point de vue dominant dans les enquêtes portant sur l'échange électoral qui s'intéressent, d'un côté, à ce que les groupes d'intérêt donnent (notamment de l'argent) et, de l'autre, à ce qu'ils reçoivent (des droits d'accès aux institutions par exemple). Nous souhaitons, pour notre part, penser ensemble les pratiques des «donneurs» et celles des « receveurs», ce que la recherche n'aborde encore qu'en creux. Pour ce faire, nous proposons d'emprunter le cadre anthropologique du don contre-don de Marcel Mauss, qui n'est pas mobilisé dans l'étude des groupes d'intérêt. Parmi les nombreuses réflexions qu'il a suscitées, celle de Paul Veyne nous permet de repenser l'échange électoral.

La focalisation sur l'influence nous semble témoigner d'un autre parti-pris de la recherche qui tend à réduire le positionnement des groupes en campagne à la structuration de «l'offre politique ». En ouvrant l'analyse de l'élection présidentielle française à ces groupes nous entendons élargir la perspective en intégrant l'ensemble de leurs pratiques en temps électoral, telles qu'elles se déploient des deux côtés de la scène électorale. Il s'agit ainsi d'ajouter notre pierre à l'édifice de la recherche états-unienne en tâchant de montrer dans quelle mesure les enjeux médiatiques et des pans de programmes des candidats sont coproduits, mais également d'envisager la participation des groupes en campagne à la structuration de «la demande» - en étudiant par exemple comment les propositions s'élaborent puis circulent jusqu'aux candidats, ou comment l'« opinion », via des sondages, est l'objet de commandes précises de la part de certains groupes. Analyser la participation des groupes en campagne des deux côtés de la scène électorale nous invite à être attentifs aux relations sociales dans lesquelles leurs membres s'insèrent et aux rites électoraux qu'ils contribuent à rendre vivants et possibles. Penser le rôle et les pratiques des groupes en campagne dans le vécu de l'élection nécessite ainsi de rendre compte de leurs tentatives de participation à la production des enjeux et des programmes politiques, quitte pour certains à s'en attribuer une part léonine et, pour d'autres, à en être exclus ou à y être censurés. Cela impose également de vérifier comment ils tentent d'exprimer des intérêts et des revendications, les plus nombreux n'étant ni compris dans «l'offre» politique ni reconnus comme la « demande». 
Ces déplacements nous invitent à entrer dans le vécu de la campagne en embrassant l'ensemble des pratiques qui s'y déploient. Cette démarche distingue notre enquête des travaux existants qui se focalisent sur l'influence et la monétarisation. Elle implique de se pencher sur la dimension collective de l'élection, ses rituels et les dimensions informelles et verbales ${ }^{4}$ des échanges qui y prennent place. Les formes élémentaires de vie collective ont en effet été "enterrées vivantes ${ }^{5}$ », avec les classes sociales, dans l'analyse électorale - vivre l'élection en famille ${ }^{6}$, dans les groupements primaires, ou au sein de groupes sociaux ${ }^{7}$ par exemple. Or l'enquête de terrain a montré que le rassemblement des membres d'un groupe au moment où une proposition est solennellement rendue publique, avant d'être envoyée aux candidats, fait partie des rites de campagne. De ce point de vue particulier sur la campagne, le rituel qui entoure la publicisation du don permet de vivre l'élection en groupe. D'autres façons de vivre collectivement l'élection seraient à prendre en compte et il conviendrait de découvrir les différentes façons de se regrouper pour discuter de la campagne. En intégrant les groupes en campagne à l'analyse de la scène électorale, nous proposons ici de reprendre l'approche classique du vote en groupe(s), tout en insistant sur l'échange verbal et le mode collectif de production des opinions ${ }^{8}$. Des citoyens vivent des séquences de la campagne au sein de collectifs, parlent des enjeux en groupe, pendant que, du MEDEF à la CGT, les groupes sont parlés et exposés dans l'espace public.

\section{Le don : la chose donnée et l'acte de donner}

Selon Paul Veyne, l'analyse des dons doit distinguer «la chose donnée et l'acte de donner ${ }^{9}$ ». Notre cadre d'analyse entend d'abord rendre raison à la variété des « choses données » et éclairer la façon dont les programmes et les prises de position sont co-

4Sur le vote de groupe et les analyses de la conversation lors des élections, voir BRACONNIER C., Une autre sociologie du vote. Les électeurs dans leurs contextes: bilan critique et perspectives, Cergy Pontoise, Lextenso éditions, coll. « LEJEP », 2010.

5LEHINGUE P., Le vote. Approches sociologiques de l'institution et des comportements électoraux, Paris, La découverte, coll. « Grands repères Manuel », 2011, p. 260.

6Voir le protocole expérimental suivi par l'équipe de Chistophe Le Digol lors de l'élection présidentielle de 2012. Journée d'études autour des travaux SPEL, Université Paris 1 Panthéon-Sorbonne, Centre Malher, 14 septembre 2012.

7Sur la religion catholique, voir Deloye Y., Les voix de Dieu. Pour une autre histoire du suffrage électoral : le clergé catholique français et le vote- $X I X^{e}-X X^{e}$ siècle, Paris, Fayard, coll. " L'espace du politique », 2006. 8BouRdiEU P., " "Le mystère du ministère" ; Des volontés particulières à la "volonté générale" », Actes de la recherche en sciences sociales, $\mathrm{n}^{\circ} 140,2001, \mathrm{p} .7-11$.

9VEYNE P., Le pain et le cirque. Sociologie historique d'un pluralisme politique, Paris, Le Seuil, coll. « Points Histoire », 1976, p. 81 et $s q$. 
fabriqués par les groupes en campagne et les partis politiques. Aux États-Unis, l'analyse de la coproduction de la campagne électorale passe par celle des contributions financières. Or limiter la théorie de l'échange et le don contre-don aux seuls échanges monétaires revient à créer un angle mort dans l'analyse de la scène électorale. Si les élections étudiées dans cet ouvrage n'ont pas fait l'objet de contributions financières connues, cela n'empêche pas certains groupes d'avoir beaucoup dépensé pendant la campagne - les budgets les plus importants atteignant 500000 euros $^{10}$. Les « choses données » renvoient ici à une large palette de produits électoraux ${ }^{11}$, dont beaucoup sont des biens symboliques (arguments, slogans, idées, propositions, projets, plateformes, solutions, informations ${ }^{12}$, expertises ${ }^{13}$ ) et parfois des biens matériels (camions, cars, salles, matériel technique), voire du capital social. Les dons de campagne, au sens anthropologique du terme, permettent aux candidats de disposer d'un stock de ressources pour parler à la presse, élaborer un discours ou aménager un programme. Une large part des biens politiques proposés aux électeurs est ainsi le produit de cet échange et ne peut se comprendre sans intégrer le rôle que les groupes en campagne ont joué dans leur élaboration, et parfois dans leur usage.

Ce cadre anthropologique permet de dépasser la conception restrictive du don comme recouvrant seulement des agrégats financiers et rend possible des analyses sur l'échange électoral non pécuniaire dans le cas français. Ouvrir de la sorte l'analyse de l'échange électoral ne présume pas de la réussite des groupes en campagne dans cette interaction. Les enquêtes convergent pour souligner les biais et censures développés dans le champ politique pour exclure certaines demandes de la composition finale des biens électoraux, pendant l'élection comme durant la session parlementaire, ou pour ne pas autoriser certains groupes à participer à la production de ces mêmes biens. L'inégale

10Notons néanmoins que les informations relatives aux budgets consacrés à la campagne électorale sont difficilement accessibles. Ce budget maximal a été trouvé à deux reprises au cours de notre enquête, dans le cas d'une ONG environnementale et dans celui de la branche française d'une organisation internationale.

11Voir leur mise en perspective dans LeHINGUE P., Le vote...., op. cit., p. 43.

12de Figueiredo J. M., «Lobbying and Information in Politics », Business and Politics, 4 (2), 2002, p. $125-129$.

13P. Zittoun analyse la transformation de la politique du logement en insérant l'expertise de ce domaine dans une relation d'échange (entre experts et partis politiques) dans le cadre des élections présidentielles de 1981 et 1995 et des législatives de 1993. Il postule ainsi que l'on peut tracer la trajectoire d'un argument et en attribuer la paternité. En l'espèce, les programmes politiques ne font que reprendre des propositions «déjà formulées dans des rapports d'experts ». En échange de ces ressources, les partis établiraient une dette qu'ils s'engageraient à rembourser «s'ils sortent vainqueurs des élections» («Partis politiques et politiques du logement. Échange de ressources entre dons et dettes politiques», Revue française de science politique, 51 (5), 2001, p. 684). 
répartition des ressources et du savoir-faire entre les groupes en campagne, ainsi que leur maîtrise différenciée des règles du jeu politique orientent également l'acte de don.

Si la recherche états-unienne a beaucoup quantifié et analysé le contenu de l'échange électoral ( «la chose donnée ») entre candidats et groupes d'intérêt, l'acte lui-même n'est pas interrogé et paraît aller de soi. Cette posture repose sur un scénario d'anticipation de l'avenir - la fameuse préemption du droit d'accès - qui l'emporte sur la prise en compte de ce qui se déroule dans le temps présent par «l'acte de donner ». L'absence d'analyse de cet acte jette un voile sur toutes les pratiques qui accompagnent le don électoral. Candidats et groupes font-ils d'autres choses ensemble durant la campagne et en dehors du temps électoral ? Cette importante question demeure en demi-teinte dans nombre de travaux qui réduisent ces «largesses» à «un prix d'achat des consciences $^{14} »$. Les actes permettant de donner sont variés : aux simples envois de courriers postaux ou électroniques s'ajoutent des cérémonies plus ou moins ritualisées et plus ou moins publiques, lors desquelles des candidats sont parfois invités à signer des textes en témoignage de leur engagement. Observer ces scènes permet de saisir ce que la focalisation sur la seule "chose donnée » occulte; par exemple, à quel point l'échange impliqué repose a priori sur un aléa, la rencontre entre le bien offert et la personne censée le recevoir n'étant pas garantie. Dans de nombreux cas, l'observation montre que la «chose donnée» existe mais que «l'acte de donner» a été imparfaitement accompli, ou sans conviction ni volonté manifeste du donateur ou de la donatrice. Par exemple, des groupes en campagne donnent sans même identifier ou nommer le, la ou les receveurs; d'autres mettent en ligne des lettres ouvertes qu'ils négligent d'envoyer à ceux auxquels ils s'adressent; d'autres encore (par méconnaissance des règles du jeu politique) écrivent aux candidats la semaine du premier tour ; d'autres, enfin, se contentent de publier des communiqués de presse sur leur site, sans s'assurer de leurs diffusions. Nombre de permanents semblent avoir intériorisé la croyance selon laquelle tous les candidats et leurs équipes seraient à l'écoute, voire en quête, de tout ce qui est énoncé à leur intention, même si le « don » s'est opéré sans acte ou contact.

L'observation in situ de l'«acte de don», lorsqu'elle appréhende ceux qui donnent et ceux qui reçoivent, permet également de montrer la pluralité de personnes impliquées

14Ibid., p. 380. 
derrière ces deux figures ${ }^{15}$, souvent présentées de façon monolithique. Le camp des donneurs est symbolisé par une organisation qui peut prendre des formes différentes un syndicat, une association, une organisation professionnelle, un think tank- et s'incarne dans des personnes singulières - le responsable, la présidente, des regroupements d'individus, comme une assistance dans un théâtre par exemple. Le camp des receveurs est lui aussi un dégradé de figures : une candidate sur scène, les candidats qui se succèdent, ou certains d'entre eux seulement, la conseillère ou le représentant de la candidate, ou encore la personnalité parlant au nom de la candidate.

Ce cadre anthropologique et l'attention portée à l'acte de don lui-même donnent enfin à voir la possible dimension collective de l'acte de don. Les rares scènes de collectes de dons financiers étudiées aux États-Unis impliquent des personnes qui se connaissent largement toutes, et quand l'élu ou la candidate tardent à remettre l'une d'entre elles, un membre de leur staff se doit d'intervenir aussitôt pour éviter l'impair ${ }^{16}$. Buffets, repas, petits déjeuners sont ainsi le cadre d'interactions permettant également de saisir si la sociabilité manifestée objective ou renforce des affinités idéologiques et sociales. En somme, la dichotomie de P. Veyne permet d'élargir et d'affiner dans le même temps notre compréhension des biens électoraux offerts. Elle nous invite à rendre raison du don en actes, que celui-ci ait lieu en public, dans le cadre de cérémonies ouvertes à tous, ou qu'il se déroule de manière plus confidentielle, dans le QG des candidats, sans médias ni spectateurs.

\section{Les effets symboliques de «la chose donnée »}

Le don électoral produit des effets symboliques de part et d'autre: financer ou être financé est un signe de distinction électorale. L'insertion des acteurs de l'élection dans une relation d'échange permet de mettre en lumière les potentialités qu'une telle relation ouvre pour chacun·e des protagonistes.

Pour les candidats, les sommes obtenues sont un gage de crédibilité. Aux États-Unis, être compétitif nécessite de pouvoir acquitter un ticket d'entrée sur le marché électoral

15Rappelons que, chez Mauss, ces deux figures sont plurielles et renvoient à des groupes ou à des sousgroupes.

16Voir la note de terrain qui ouvre Francia P. L., Green J. C., HerRnson P. S., Powell L. W., WiLCoX C. (dir.), The Financiers of Congressional Elections. Investors, Ideologues and Intimates, New York, Columbia University Press, 2003. 
calculé autour de 800000 dollars ${ }^{17}$. Avant même le vote, et sans tenir compte d'aucun sondage, le budget collecté est donc l'indicateur le plus sûr de l'échec politique annoncé18. Ce budget participe ainsi de la transmutation d'un·e candidat.e en challenger, d'un·e sortant.e en defender. Avantage comparatif indéniable par les moyens qu'il met à disposition pour mener une véritable campagne, le budget laisse également présager un pourcentage de votes impossible à évaluer réellement. Pour un·e challenger, il faut pouvoir dépenser plus que la moyenne pour l'emporter. Pour un·e sortant·e, il faut avoir un budget permettant de faire face à une contestation d'autant plus forte que l'adversaire détient un budget de challenger. Dans cette logique, certains seuils de financement équivalent à une probabilité de conserver son mandat ou de remporter l'élection. L'analyse des dépenses montre que les gagnants sont ceux parmi les candidats qui ont le plus dépensé. Cela revient à souligner que, en dehors des rares circonscriptions où la compétition est disputée, les sortants dépensent plus que les challengers - le taux de réélection moyen pour les élections à la Chambre des représentants depuis 1945 est de $91 \%{ }^{19}$.

Du côté des donneurs, quelles sont les formes de croyance qui animent les groupes en campagne? Sur ce terrain également, l'analyse des dons funéraires proposée par P. Veyne est un guide utile. Ces dons se combinent à une mythologie permettant d'imaginer que la nourriture déposée sur une tombe vient nourrir des défunts. L'emprise d'un même "programme de vérité20 » dans l'accomplissement de l'acte électoral, voire dans l'analyse des produits électoraux, permet d'imaginer qu'une liste de douze propositions « offerte » par un groupe durant la campagne sera utilisée, dans une autre arène ou dans un autre contexte, pour élaborer un programme ou pour rédiger une loi quelques années après. Marcel Mauss insiste, lui, sur les effets symboliques des dons et montre que l'honneur des participants et de leur groupe d'appartenance est en jeu, ainsi que leur rang dans la société. Les sommes offertes relèvent d'une forme de

17Jacobson G. C., The Politics of Congressional Elections, New York, Pearson Longman, 2009 [1 ère éd. 1983], p. 47.

18En France, ceux qui dépensent le plus sont issus des partis les plus importants et obtiennent généralement le plus. Voir l'analyse des comptes de campagne faite par LEHINGUE P., «Les budgets des partis et des campagnes électorales comme matériau sociologique: opportunités et limites de l'objectivation comptable » in E. PHELIPPEAU, A. FRANÇOIS (dir.), Le financement de la vie politique française. Des règles aux pratiques (1988-2009), Paris, éditions Weka, 2010, p. 23-48.

19MARREL G., PAYRE R., « Des carrières au Parlement. Longévité des eurodéputés et institutionnalisation de l'arène parlementaire ", Politique européenne, 18, hiver 2008, p. 95.

20Veyne P., Les Grecs ont-ils cru à leurs mythes? Essai sur l'imagination constituante, Paris, Le Seuil, coll. «Points Essais », 1983. 
magie sociale en venant conforter la «grandeur» de certains. Acte d'homologation, le don financier consacre ainsi la participation de certaines entreprises à la «vie démocratique» et leur octroie officiellement le statut d'institution civique. Le financement des campagnes vient également attester leur notoriété durant l'élection, au même titre que l'occupation d'un bureau de représentation à Washington est un gage de leur puissance économique hors temps électoral ${ }^{21}$. La transparence sur les comptes de campagne étant de mise, la presse et les chercheurs états-uniens citent régulièrement les plus grands financeurs, consacrant par là une part de ceux qui donnent pour faire élire ${ }^{22}$. C'est certainement une explication de la gradation des investissements réalisés en campagne. Mais tous les groupes ne disposent pas des ressources suffisantes ou d'un sens pratique adapté - ou suffisamment développé - pour utiliser tout ou partie des pièces du répertoire en campagne et pendant la session parlementaire. À ceux qui peuvent jouer dans les deux séquences en utilisant, pour chacune, les formes d'action les plus légitimes (produire des biens électoraux et entretenir une équipe permanente de relations institutionnelles) s'opposent ceux, plus nombreux encore, qui ratent leurs cibles ou ne parviennent pas à atteindre les objectifs qu'ils se fixent.

Les effets symboliques du don et les assignations de rôle qu'il produit ne sont pas toujours positifs. Car la division électorale du travail politique est productrice de rôles que le don, dont les montants sont officialisés par les institutions, permet d'homologuer. Maurice Godelier précise ainsi la pertinence du cadre du don contre-don en montrant que l'interaction maintient et renforce la distance et la différence entre les deux groupes qui échangent ${ }^{23}$. Cet échange implique une « dissymétrie, une hiérarchie, entre celui qui donne et celui qui reçoit ${ }^{24} »$. Si, chez Mauss, le don place le donateur ou la donatrice dans une position de supériorité, cette position n'est pas nécessairement ni toujours la plus valorisée socialement, notamment lorsque ces pratiques sont dénoncées. Ainsi les «donneurs » sont souvent astreints au «mauvais » rôle soit, en termes sociologiques,

21SAlisBuRY R. H., « The Paradox of Interest Groups in Washington. More Groups, Less Clout » in A. KING, (dir.), The New American Political System, Washington, American enterprise institute for public policy research, 1990, p. 220.

22Les travaux citent souvent telle ou telle entreprise. Par exemple, M. Franz retient qu'en une année électorale W. Disney a donné à peu près un million de dollars de soft money alors qu'il y a eu 508 autres donneurs. Franz M. M., Choices and Changes. Interest Groups in the Electoral Process, Philadelphia, Temple University Press, 2008, p. 21.

23Godelier M., Au fondement des sociétés humaines. Ce que nous apprend l'anthropologie, Paris, Albin Michel, 2007, p. 70.

24Ibid. 
celui dont on revêt ceux qu'on accuse de transgresser les normes en vigueur. Cette accusation frappe rarement au hasard et elle est très souvent empreinte d'ethnocentrisme, désignant comme «vulgaire» celui ou celle qui transgresse les conventions et autres prescriptions de l'étiquette en politique - parler d'argent ou n'en pas faire mention, n'évoquer que son intérêt ou taire ceux qui sont censés être prioritaires. Aux États-Unis, certains candidats interrogés sur la collecte de fonds en parlent ainsi comme la partie la moins agréable de la campagne, et expriment parfois même leur « dégoût ${ }^{25}$ » vis-à-vis de certaines activités, telles que le « dial for dollars » ou « talking to strangers ${ }^{26}$ ». Ils se défaussent alors de ces activités considérées comme peu valorisantes, voire dégradantes, sur des groupes en campagne. Rationnelle à plus d'un titre, cette division du travail permet au personnel politique d'économiser une partie de son temps et de bénéficier du capital social des autres. L'analyse de l'échange électoral montre que le choix par un·e candidat·e d'un groupe auquel déléguer la collecte de fonds n'est pas nécessairement orienté par les caractéristiques économiques de l'organisation en question (cotisations des membres, fortunes personnelles des dirigeants et contributions des soutiens) mais peut être déterminé par le capital social de celle-ci, ainsi que sa position dans le champ. Plus généralement, la situation de dépendance financière et logistique des partis politiques doit s'appréhender par l'introduction d'autres critères que les seules variables économiques ${ }^{27}$.

Iconographie de la campagne présidentielle : rencontres entre des groupes, les candidats et leurs équipes. Ces photographies sont publiées par les groupes en guise de témoignage de leur influence. Elles attestent également 1' expertise de certains membres des équipes de campagne sur des enjeux précis.

25Ce ressenti est souligné par WiLhite A., Theilmann J., «Unions, Corporations, and Political Campaign Contributions: the 1982 House Elections », Journal of Labor Research, 7 (2), 1986, p. 176-177. Il fait écho aux autres «sales boulots » de la campagne laissés par les dominants aux subalternes. La dévolution du sale boulot aux dominés est un classique de la sociologie des professions, souligné par E. C. Hughes dans : Le regard sociologique. Essais choisis, Paris, EHESS, 1996.

26FranciA P. L. et al., The Financiers of Congressional Elections, op. cit., p. 78.

27Cette situation de dépendance renvoie à: GAXIE D., «Économie des partis et rétributions du militantisme », Revue française de science politique, 1977, 27 (1), p. 123-154) qu'il faut étendre aux organisations impliquées dans l'échange électoral, soit en explorant les effets des ressources personnelles des candidats sur leur besoin de financement (nombre d'auteurs états-uniens soulignent ainsi le plus faible volume de moyens financiers du Parti démocrate dont les leaders sont moins fortunés que les Républicains), soit en analysant la dépendance quasi structurelle d'un parti vis-à-vis de soutiens extérieurs (les syndicats liés à des partis, ou certains grands patrons ou certaines organisations professionnelles). 

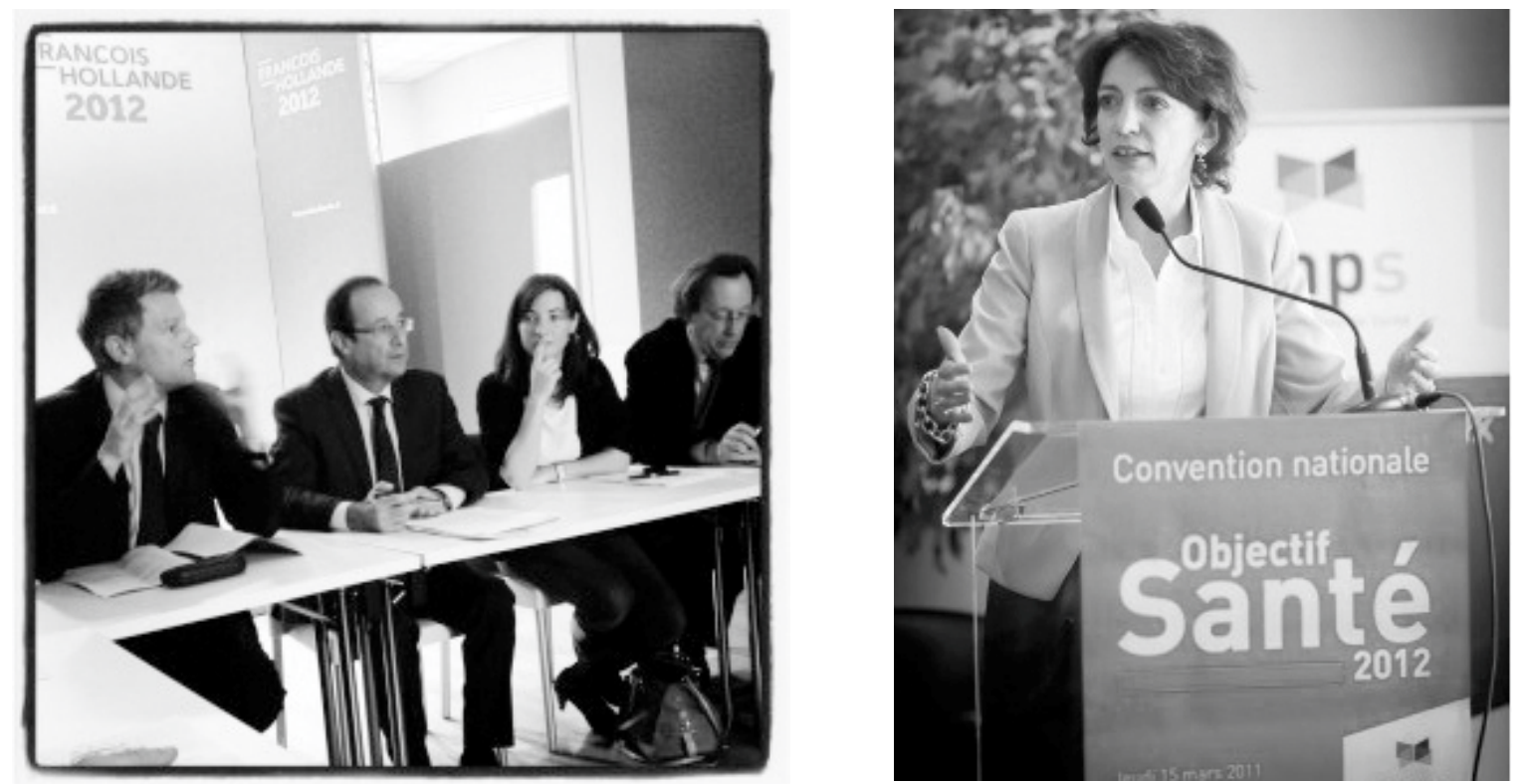

À gauche, photographie exemplaire des rencontres organisées dans les QG de campagne, prise lors de la réception par le candidat F. Hollande et une partie de son équipe (dont V. Peillon, futur ministre de l'Éducation, à sa droite) des douze organisations cosignataires de la plateforme 《Pour un big-bang des politiques jeunesse ! ${ }^{28}$.

À droite, photographie illustrant le rituel électoral du 《 grand oral 》 avec des représentants des candidats

(ici M. Touraine, future ministre de la Santé de F. Hollande), organisé par le Centre national des professions libérales de santé lors de la présentation de leur plateforme 《2012, le pari de la santé 》.

\section{Le contre-don : I'obligation, la chose, ses rituels}

Dans son Essai sur le don, M. Mauss montre combien le don oblige le groupe ou le sousgroupe qui le reçoit et qui se trouve ainsi placé dans la nécessité de se libérer par une « contre-prestation ${ }^{29}$ ». Mais, contrairement à ce qui est attendu dans les sociétés qu'il qualifie d'«archaïques", le don accompli en campagne électorale n'entraîne pas nécessairement de retour sous la forme d'un contre-don.

Les travaux menés sur les financements de l'élection par les groupes d'intérêt présentent des conclusions convergentes quant à leurs effets politiques : selon elles, il

28Ces cosignataires sont: CNAJEP (jeunesse et éducation populaire), FNARS (accueil et réinsertion sociale), UNIOPSS (secteur non lucratif de solidarité), FNMF (mutualité française), UNML (missions locales), JOC (jeunesse ouvrière chrétienne), MRJC (jeunesse rural chrétienne), UNHAJ (habitat des jeunes), ANACEJ (conseils de jeunes), ANMDA (maison des adolescents), CNLAPS (prévention spécialisée), GNDA (secteurs éducatif, social, médico-social). Voir le site http://www.bigbangjeunesse.net 29MAuss M., "Essai sur le don. Forme et raison de l'échange dans les sociétés archaïques », L'Année sociologique, nouvelle série, t.1, 1923-1924, p. 31 et sq. Mauss n'utilise pas le terme de « contre-don » qu'il ne cite qu'une fois (p. 80) en l'empruntant à B. Malinowski. 
n'y aurait pas d'effet direct des financements de la campagne sur les biens politiques obtenus après l'élection, que ces biens soient des textes juridiques, des votes, voire des amendements ou des questions parlementaires ${ }^{30}$. La question du pré-achat des votes a fait l'objet d'un protocole de recherche suivi par Richard A. Smith ${ }^{31}$. Les résultats précisent que huit conditions président à la relation causale entre le fait d'avoir financé et celui d'obtenir un vote favorable d'un ou d'une élue. Elles se ramènent toutes à des enjeux sans visibilité, sans opposition, non politisés, sans répercussion fiscale pour les citoyens, n'ayant pas d'impact sur l'opinion, ne concernant qu'une seule organisation, et ayant fait l'objet d'un travail du groupe concerné et de celui d'une commission parlementaire. Une telle réduction de l'arène interroge sur la possibilité d'avoir beaucoup de cas de cette sorte à observer. En suivant la carrière politique de deux enjeux, J. Wright montre à son tour que ce n'est pas un vote particulier qui est attendu en retour d'un don mais un accès qui est ainsi préempté32. La question de la préemption du droit d'accès est disputée. Longtemps, la loi de l'achat d'un tel droit ${ }^{33}$ n'a été testée que sur les $P A C^{34}$ (voir le chapitre 1). Pour les uns, l'effet serait quasi automatique, la contribution financière suffisant à prouver la préemption. Pour les autres, l'effet serait relatif aux enjeux concernés : il serait ainsi nul, ou moindre, sur les enjeux saillants porteurs de clivages idéologiques, et beaucoup plus net sur les enjeux moins visibles et

30Une recherche originale a porté sur la mise en relation des sommes reçues par les candidats avec les questions posées par les élus lors des auditions parlementaires des groupes concernés. Elle montre que si les deux sont indissociables - ceux qui interrogent le plus sur les questions de santé sont ceux qui sont le plus financés par les $P A C$ concernés - il s'agit plus d'une démonstration supplémentaire de la logique de spécialisation du travail politique que de la preuve d'une dérive du système parlementaire. ESTERLING K. M., «Buying Expertise: Campaign Contributions and Attention to Policy Analysis in Congressional Committees », American Political Science Review, 101 (1), 2007, p. 93-109.

31Smith R. A., «Interest Group Influence in the U.S. Congress », Legislative Studies Quarterly, 20 (1), 1995, p. 94.

32Wright J. R., Interest Groups and Congress. Lobbying, Contributions, and Influence, New York, Longman, 2003, p. 430. Son étude ne lui permet pas de conclure à l'efficacité des contributions financières sur les votes à venir des parlementaires pendant la législature car ce sont les variables « partis » et « idéologies » qui sont les plus efficientes pour les expliquer. J. R. Wright développe ainsi une analyse originale et pertinente : les candidats « ajustent leurs positions électorales pour attirer l'argent » (ibid., p. 148) et ils promettent un accès à leurs bureaux parce qu'ils n'ont pas le temps de recevoir tous les lobbyistes pendant la campagne.

33Elle a été formulée initialement par HANSEN J. M., Gaining Access. Congress and the Farm Lobby, 19191981, Chicago, Chicago University Press, 1991 ; elle est reprise et généralisée par SMITH R. A., «Interest Group Influence... », art. cit., p. 93. Voir également la synthèse sur les PAC de SmITH M. A., American Business and Political Power. Public opinion, Elections and Democracy, Chicago, The University of Chicago Press, 2000, p. 119-125.

34LAngbein L. I., « Money and Access : Some Empirical Evidence », The Journal of Politics, 48 (4), nov. 1986, p. 1060. 
plus techniques ${ }^{35}$. L'enquête de Peter L. Francia et al. ${ }^{36}$ indique quant à elle que, très souvent, les groupes d'intérêt n'attendent aucune faveur particulière en retour de leurs contributions financières.

Quand les chercheurs montent en généralité, la formule la plus fréquemment utilisée est que les groupes d'intérêt espèrent avoir acquis un droit d'entrée, une sorte de police d'assurance ${ }^{37}$. Pour valider une telle montée en généralité au regard de l'hétérogénéité des groupes concernés, encore faut-il néanmoins passer d'une réflexion sur une relation causale entre des pratiques à l'exploration de l'univers de croyances des donateurs. Cet espoir émaille en effet les propos des donateurs interrogés qui disent avoir une chance de «faire entendre leur voix ${ }^{38}$ », de «donner leur opinion sur une loi ${ }^{39}$ » et autres formules plus ou moins propitiatoires. La campagne électorale est donc le moment où se préempte, peut-être, le droit d'avoir l'espoir d'exercer le métier de représentant·e d'intérêt dans le champ politique. Elle est certainement le moment où un tel espoir s'énonce, surtout si cela permet de faire état des produits électoraux qui ont fait l'objet d'un don à un·e candidat·e. L'évergétisme, insiste P. Veyne, est une relation sociale en soi avant d'être une fin ${ }^{40}$. En somme, le don peut-être l'expression d'un droit à la parole et non la revendication d'un droit à l'écoute.

Adopter le cadre anthropologique du don contre-don permet également de se pencher sur ce que recouvre la chose rendue. Tous les biens politiques peuvent-ils ainsi faire l'objet d'un échange? M. Godelier montre que certains biens sont hors de l'échange: dans la vie quotidienne, cela peut notamment être le cas des biens qui sont exclusivement en jeu lors de l'héritage. La recherche consacrée aux clientélismes et aux mobilisations électorales insiste sur l'échange ancien, continu et classique d'une gamme étendue et variée de biens entre des candidats et des électeurs potentiels. Mais la question reste entière sur les biens politiques indivisibles. La loi se situe-t-elle dans l'échange ou hors du marché politique? Quid des politiques publiques? Certaines d'entre elles demeurent-elles en dehors du cadre de l'échange ? Notre enquête indique

35Smith M. A., American Business..., op. cit., p. 125.

36FrAnCIA P. L. et al., The Financiers of Congressional Elections, op. cit.

37Baumgartner F. R., Berry J. M., Hojnacki M., Kimball D. C., LeECh B. L., Lobbying and Policy Change, Who

Wins, Who Loses, and Why, Chicago, Chicago University Press, 2009, p. 193.

38Francia P. L. et al., The Financiers of Congressional Elections, op. cit., p. 124.

39Ibid., p. 127.

40VEYNE P., Le pain et le cirque..., op. cit., p. 81 et $s q$. 
par exemple que certains candidats refusent toute concession ou intervention exogène sur certains thèmes - notamment en fonction de leur domaine de spécialisation et de leur camp idéologique - quand d'autres consentent au marchandage alors qu'ils ne sont crédités d'aucune chance d'être élus.

Qu'elle intervienne effectivement ou pas, la potentialité d'une contrepartie permet d'inscrire l'échange électoral dans la longue durée, le contre-don étant nécessairement décalé dans le temps. Des allers retours sont alors possibles entre ce qui a eu lieu en campagne et ce qui se joue en session. Une fois le contact établi et les habitudes ou préférences d'un·e candidat·e repérées ${ }^{41}$, des groupes en campagne vont le ou la pousser pour en faire «leur candidat.e». Une fois le résultat de l'élection connu, le donateur ou la donatrice pourra rappeler à ses obligations le ou la nouvelle élue, essaiera de lui fixer certaines orientations en fonction de ses promesses, de cadrer ses interventions ou de prioriser ses actions selon les objectifs convenus en campagne.

Dans ses amendements à l'universalisme du don contre-don, M. Godelier précise que l'acte de don doit être suivi d'une chaîne d'actes pour susciter le contre-don. Une sorte de déclinaison de pratiques d'accompagnement jouant à la manière de «conditions de félicité42 »; un ensemble cohérent d'actes à accomplir et de formules à prononcer pour mettre celui ou celle qui reçoit dans l'obligation d'introduire un contre-don dans l'échange. En passant trop rapidement du donneur aux pratiques du receveur, on oublie de montrer comment les donateurs vivent le don, le préparent, le justifient, le valorisent et tentent de contrôler ou a minima de suivre ses potentiels effets. En pensant intuitivement que l'adoption par les pouvoirs publics (le nouveau gouvernement ou la nouvelle majorité) d'un texte réglementaire ou législatif reprenant la demande énoncée est la contrepartie du don, nous n'avons à l'esprit qu'une vision fragmentaire et incomplète de la relation d'échange. À supposer en effet que ces biens soient échangeables, le contre-don s'insère dans toute une chaîne d'actes qui interviennent après le don, rien n'indiquant que ces actes doivent avoir lieu pendant la campagne. Retracer la carrière d'un enjeu et les pratiques de ceux qui le portent met en lumière de

41Certains lobbyistes tiennent ainsi des tableurs dans lesquels ils consignent les données politiques, biographiques et sociodémographiques des candidats comme, mais plus rarement, leurs goûts ou traits de caractère les plus connus.

42Ce sont ces conditions qu'explore Austin pour comprendre comment des mots peuvent faire faire quelque chose. Austin J. L., Quand dire, c'est faire, Paris, Le Seuil, coll. « Points Essais », 1970. 
nombreux actes, apparemment anodins, qui s'égrènent après l'envoi des propositions. Ces actes vont de la réitération d'une proposition pendant l'entre-deux tours, adaptée aux deux seuls candidats en place, aux nombreux emails, textos et appels auprès des conseillers. S'y ajoutent les demandes de rendez-vous « une fois que le gouvernement sera installé », les actes de reconnaissance des futurs ministres ou des futurs leaders de l'opposition, jusqu'aux lettres et appels de félicitation dès les résultats du second tour connus, ou dans la semaine qui suit au plus tard. Une campagne électorale nécessite ainsi une série d'actes qui ne peuvent se résumer à l'écriture d'un courrier ou d'une proposition. Toutes ne sont pas nécessairement adressées aux candidats et à leurs équipes et ce que d'aucuns considèrent comme de simples stratégies de conquête de l'opinion (communiqués de presse, conférences, etc.) relèvent souvent de pratiques accompagnant le don.

Tous les groupes en campagne ne sont pas concernés, loin s'en faut, et ceux qui arrivent à respecter ces conditions de félicité sont en réalité les moins nombreux. Ces actes d'accompagnement objectivent en effet l'homologie, le fort entre soi, et les interrelations qui unissent quelques acteurs de ces groupes en campagne à certains candidats ou à leurs équipes ${ }^{43}$. Il convient ainsi d'intégrer à l'analyse le travail des conseillers des équipes de campagne qui participent à l'engagement du contre-don. Des élus du parti aux relations d'un·e candidat·e, en passant par les journalistes, nombreux sont d'ailleurs ceux qui appuient, rappellent, conseillent, et discutent pour inciter à faire faire (ou non) quelque chose suite à l'acte de don d'un groupe en campagne. Après avoir ouvert l'élection aux groupes en campagne, il faudrait donc poursuivre ce travail d'élargissement de l'échange électoral en intégrant le rôle joué par ce personnel de renfort qui contribue, de fait, à stabiliser la matière des échanges durant la campagne et, parfois, à fixer certains des enjeux qui seront discutés après la campagne. Le contexte de l'échange doit également être pris en compte, et les agendas médiatiques et politiques y occupent une place centrale. On a pu voir, par exemple, un candidat interrogé lors d'une émission en direct se saisir de la question posée pour utiliser comme argument un thème souligné par le responsable d'un groupe rencontré auparavant. Le travail des équipes de campagne participe à créer les conditions de possibilité d’un échange symétrique : répondre à la question d'un groupe ne s'envisage qu'après avoir vérifié le 
taux d'audience d'un site (répondre ne se fait pas sans anticiper les retombées électorales), le nombre d'abonnés d'une revue professionnelle, la quantité ou les qualités des signataires d'un texte. C'est également la règle du précédent qui prime : il faut répondre parce que la candidate, l'autre candidat, ou son propre parti, a déjà apporté une réponse, y compris si celle-ci a été formulée lors d'une campagne précédente.

La méconnaissance de ces conditions de félicité est telle qu'on s'interdit d'analyser tout ce que doivent faire les interactants pour qu'un·e candidat.e se sente dans l'obligation de rendre. Inscrire l'échange électoral dans la bonne séquence permet de ne pas résumer l'action des groupes en campagne à la seule quête de la modification des programmes électoraux. Dans cette séquence, les pratiques des candidats apparaissent, elles aussi, sous un nouveau jour quand on les inscrits dans l'urgence et la précipitation : la promesse de faire quelque chose « à l'avenir », après avoir reçu, témoigne également du fait qu'ils n'ont pas le temps de s'en occuper en campagne. À l'espoir recherché par les uns correspond une promesse : une promesse qui équivaut moins à un engagement d'écoute à venir qu'à un traitement politique remis à plus tard.

\section{(Faire) vivre l'élection en groupe}

Avec la dimension rituelle de l'échange électoral, c'est sa dimension collective que le cadre anthropologique du don contre-don vient mettre en lumière. Si la sociohistoire du vote a pu montrer que l'on votait groupé, l'étude de la participation des groupes à l'élection présidentielle de 2012 montre que cette forme collective de la vie politique n'a pas disparu : la campagne électorale se vit encore en groupe $(s)^{44}$.

Les enquêtes disponibles insistent sur l'efficacité marginale des consignes de vote lancées, plus ou moins ouvertement, par des groupes en campagne, et la sociologie électorale, arguant de leur faible poids, laisse une place secondaire aux groupes sociaux dans l'abécédaire des variables explicatives du vote. Or ces questions n'épuisent pas à elles seules l'éventail des rôles joués par ces collectifs dans les campagnes. On aurait tort d'invisibiliser leur participation au prétexte que la question de l'efficacité de leurs

44Dans son enquête ethnographique de la vie politique dans l’Yonne, M. Abélès trouvait dans les archives de P.-E. Flandin des traces de la place des groupes organisés lors des campagnes législatives de l'entredeux-guerres. Le candidat sortant était interpellé « sur son attitude à l'égard des bouilleurs de cru » et des associations promettaient leur soutien par écrit, promesse renouvelée lors de réunions électorales. Voir Jours tranquilles en 89 : ethnologie politique d'un département français, Paris, Odile Jacob, 1989, p. 85-86. 
consignes de vote sur leurs membres apparaît difficilement soluble. En effet, certains citoyens auront eu davantage de rapports à la campagne dans et parmi des groupes mobilisés que par l'intermédiaire des partis politiques. Avant d'être confrontés à un bulletin le jour du vote, ces citoyens peuvent être en présence de candidats lors de débats, rencontrer des membres d'associations ou de syndicats lors de discussions sur les programmes, ou participer à des forums sur des projets de société. Le don de biens symboliques aux candidats s'accompagne également d'une intense vie collective du fait, par exemple, des séances nécessaires à l'élaboration des demandes qui leurs sont adressées, et des réunions organisées pour en suivre la diffusion ou la médiatisation. Une fois encore, il convient de laisser de côté le moment du vote et les structures partisanes qui retiennent encore beaucoup l'attention académique pour déplacer le regard sur la campagne. À cette condition, il est possible de voir comment des groupes mobilisés participent à faire vivre l'élection à des citoyens, selon des degrés variables de densité et d'intensité.

Dans « Le mystère du ministère », courte synthèse de ses réflexions sur la délégation et sur l'opinion, Pierre Bourdieu, à la suite d'Émile Durkheim, invite à se pencher sur les conditions sociales de production d'un vote ou d'une opinion, inséparables selon lui de l'existence du groupe social au sein duquel ou par lequel ils sont produits ${ }^{45}$. Il distingue ainsi deux modes de production des opinions: l'un «atomistique et agrégatif» correspondant à la vision libérale d'une « addition mécanique des préférences »; l'autre, « collectif», qui renvoie à la campagne comme une séquence où les membres d'un groupe retrouvent et reconsidèrent « le principe de [leur] rassemblement». C'est cette deuxième dimension que nous invitons ici à explorer davantage dans le cadre de travaux universitaires.

Les personnes mobilisées vivent, un temps, la campagne sur un mode collectif en faisant ensemble ce qu'elles ne feraient pas seules: elles prennent contact avec les héros de l'histoire politique racontée par les médias. Faire vivre le groupe pendant l'élection et/ou vivre l'élection en groupe sont les deux polarités qui animent les acteurs de ces groupes. Faire vivre le groupe en agissant auprès des pouvoirs publics est un classique de l'analyse de ces organisations. Faire vivre le groupe s'inscrit dans la continuité de ce

45BouRdiEU P., « Le mystère du ministère », art. cit., p. 8. Les citations suivantes sont tirées des pages 9 et 11 du même article. 
qu'il a vécu auparavant, de ce qui lui a été dit, de ce qui a été discuté en interne. Ce « faire vivre » s'inscrit également dans une projection à plus ou moins long terme. Entre la prévision de ce que pourrait être le groupe et ce qu'il devrait être, les permanents chargés de sa maintenance ont pour mission d'accorder la sociodicée à cette nouvelle conjoncture où les mots, les slogans et les thèmes énoncés sont autant de marqueurs identitaires choisis pour convaincre les membres du bien fondé de leurs activités en campagne. Comme y invite R. H. Salisbury, il faut savoir inscrire les profits retirés d'une action politique dans la bonne économie ${ }^{46}$ : celle du leader d'un groupe qui fait signer un pacte à des candidats assure une durée supplémentaire à son contrat; celle de l'adhérent·e qui vient voir et entendre ce qui se dit bénéficie des retombées d'une partie de ce que son groupe a produit. Une part de la force symbolique du politique se niche dans ces réunions; la présence des candidats attire les membres et leur fait vivre ce qu'ils n'éprouvent que rarement ensemble: indignations, envies, émotions, huées, applaudissements scandent cette vie électorale partagée, que l'influence soit effective ou non.

$\mathrm{Si}$, pour les journalistes, ces scènes sont autant de preuves de l'influence exercée sur la campagne électorale, pour les ethnographes, elles permettent d'observer les «affinités électives » que l'étude d'une organisation ne livre pas toujours. Les cortèges, les achats groupés de publicité, les invitations à des colloques et petits déjeuners, les auditions de candidats, tout comme les signatures collectives de tracts, révèlent qui partage quoi avec qui (ou contre qui) pendant l'élection et donc, certainement, après et avant le temps de la campagne. Dans un système parlementaire comme celui de la France, le marquage des affinités ne se fait pas par l'échange monétaire mais par d'autres formules. On retrouve là le clivage entre ceux qui pensent devoir s'adresser à l'ensemble des candidats (comme ils s'adressent à l'ensemble des parlementaires), ceux qui n'entendent s'entretenir qu'avec les candidats de « leur » camp politique, ou encore ceux qui en excluent certains de façon sélective. Ainsi, lors de l'élection présidentielle française de 2012, une majorité de groupes ne s'est pas adressée aux dix candidats en lice. Et presque tous les groupes qui font partie d'un système d'action partisan n'ont obtenu de réponses que des seuls

46SAlisbuRY R. H., «An Exchange Theory of Interest Groups », Midwest Journal of Political Science, 13 (1), 1969 , p. 1-32. Cette formulation initiale portait sur la question de la création des groupes avec cette innovation qui était de centrer l'analyse sur les individus et leurs interactions. Depuis, la théorie de l'échange est davantage invoquée pour analyser les politiques publiques ou les relations de pouvoir que pour comprendre les relations entre membres des groupes. 
candidats de leurs bords. Ces logiques montrent combien l'échange varie en campagne et ne peut s'expliquer de façon simpliste par le repérage de quelques variables relatives à l'organisation ou aux textes produits par ces acteurs collectifs.

Vivre l'élection en groupe renvoie ainsi à une sorte de caléidoscope de situations dans lesquelles les produits électoraux sont présentés, discutés, amendés, débattus et finalement proposés. Dans les quinze jours qui ont précédé le premier tour de l'élection présidentielle française de 2012, Paris bruissait de multiples manifestations quotidiennes liées à la campagne. Le même jour, par exemple, ATD Quart Monde animait une soirée-débat, le syndicat CGT des agents des douanes organisait une manifestation de rue et la Confédération de l'artisanat et des petites entreprises du bâtiment tenait un congrès pour interpeller les candidats, pendant que les militants et sympathisants de Chiche! se regroupaient pour «débattre de l'environnement». Dans un lieu, la mobilisation portait sur une pétition à faire signer, dans un autre, les candidats étaient invités à débattre autour d'un thème et, ailleurs, une conférence de presse organisée dans un théâtre visait à présenter les résultats obtenus aux questionnaires envoyés début 2012 aux candidats en lice. Certaines cérémonies ont un cadre restreint au départ, élargissant par cercles successifs le nombre et la qualité des participants pour finir, parfois, par animer l'espace public ouvert au plus grand nombre - membres, sympathisants, voire simples curieux. D'autres vivent la même expérience en fondant des alliances: de la rencontre entre des visions du monde découlent d'autres propositions et autant de «contrats » de société pour le nouveau quinquennat. Dans cet éventail de situations s'observent des échanges et des flots de paroles sur les attentes, espoirs et projets pour l'après élection. Cette effervescence révèle ainsi la place centrale qu'occupent les échanges verbaux, en tant que « principes unificateurs de la situation et du groupe 47 » qui permettent de qualifier des « situations », d'offrir par là au groupe une lecture partagée de celles-ci et, en retour, de «manifester le groupe » qui peut ainsi faire corps.

Parler le groupe, discuter entre membres, dialoguer avec des journalistes et des candidats : tous ces palabres tissent le quotidien d'une campagne. En faire l'inventaire n'a pas pour objectif de montrer l'extraordinaire diversité que la conversation produit dans nos sociétés lors des périodes d'effervescence. Une autre logique sous-tend notre

47BouRdiEU P., « Le mystère du ministère », art. cit., p. 11. 
approche : nous faisons l'hypothèse qu'à l'instar du vote qui fait discuter l'électeur ${ }^{48}$, la campagne oblige les groupes à combiner la communication verbale à leur production d'écrits. Loin d'être repérable uniquement dans des supports écrits, l'influence, cette chance de faire faire quelque chose à un détenteur du pouvoir, se joue et s'observe lors des discussions qui accompagnent la production d'écrits. L'influence par temps de campagne ne s'écrit pas autant qu'elle se parle ${ }^{49}$. Là aussi, l'approche classique de l'action électorale des groupes d'intérêt mérite d'être repensée. Ces temps de la discussion sont nombreux et animent tant les groupes en campagne dans leurs préparations des demandes que les candidats dans l'élaboration de leurs réponses (ou de leur refus de répondre). Des deux côtés, avec parfois des médiateurs ou des courtiers faisant le lien, tout peut se discuter avec plus ou moins d'ardeur, de fièvre et d'énervement, selon les thèmes, le contexte et la configuration dans laquelle cela se joue. Rarement qui n'a pas discuté influe. Potentiellement, qui a systématiquement discuté de l'utilité de proposer un thème, puis de la nécessité de le médiatiser, d'en informer une équipe, d'en débattre avec elle, et lui a ensuite rappelé qu'il en a été discuté, a quelque chance d'avoir fait faire quelque chose à ces professionnels de la politique. $A$ minima le groupe concerné ne leur aura fait que prendre conscience d'un enjeu. A maxima il leur aura fourni, clés en main, la solution au problème qu'ils viennent peut-être de considérer comme urgent.

Cette proposition n'est pas teintée d'une conception angélique de la représentation des causes ou des intérêts qui, d'un seul coup, corrigerait les dysfonctionnements de la démocratie représentative. La thèse selon laquelle « qui discute a des chances d'influer » ne dépeint pas une société dans laquelle n'importe quelle cause aurait la chance d'être entendue parce qu'elle a été énoncée en présence d'un·e candidat·e. Elle ne verse pas non plus dans l'illusion de zélateurs de la « société civile» qui n'auraient pas conscience que dans les groupes jouent de concert la loi d'airain de l'oligarchie pour les dirigeants et celle de la sélection sociale des citoyens actifs pour les membres. Les groupes en campagne montrent sous un autre jour les mêmes travers de la société politique

\footnotetext{
48Voir les développements consacrés à ces recherches dans BRACONNIER C., Une autre sociologie du vote..., op. cit.

49Comme le soulignent E. Katz et P. Lazarsfeld, c'est bien la discussion, ou l'échange verbal, qui est au cœur des situations où des personnes peuvent changer d'opinion dans Influence personnelle, Paris, Armand Colin, 2008 [1 ère éd 1955]. L'influence prêtée aux médias n'est donc ni directe ni automatique. L'influence des groupes d'intérêt gagne à être repensée en adaptant ce cadre d'analyse. Voir cette proposition dans : COURTY G., GERVAIS J., « Lobbying on the French Campaign Trail: critical approaches to the study of influence», working paper, PSA, Cardiff, 25 mars 2013 [en ligne].
} 
contemporaine. D'un côté, le champ politique par temps d'élection n'est pas plus capable de faire face à l'afflux de demandes et censure ou ignore les voix discordantes dans son fonctionnement ordinaire. De l'autre, les groupes les plus divers ne peuvent faire oublier qu'ils parlent rarement au nom de tous.

En finissant cet ouvrage, nous souhaiterions encore une fois insister sur la contribution générale et nécessaire que l'étude des groupes mobilisés apporte à la connaissance d'une campagne et de ce qui se joue dans une élection. En plaidant la pertinence d'une approche comparable à l'évergétisme pour l'étude des élections contemporaines, cadre qui fait défaut à l'analyse du financement des campagnes et de l'action des groupes d'intérêt, l'objectif est de donner à voir l'élection comme la rare conjoncture permettant à des groupes d'accéder «à l'existence publique et à l'efficacité politique ${ }^{50}$ ». Or l'efficacité ne relève pas ici de la quête instrumentale d'une influence sur les candidats mais elle tient à l'opportunité qui se présente aux membres de reprendre la discussion sur le fondement de leur regroupement («produire une opinion sur la manière de

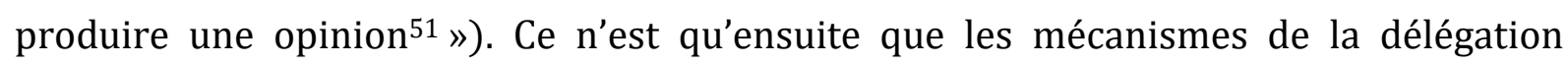
opèrent, que le ou la porte-parole dit le groupe, sa cause ou ses intérêts, et que celui-ci peut jouer le rôle qu'il aura collectivement défini en s'opposant frontalement ou en négociant bilatéralement : la quête de l'influence peut alors commencer.

50BouRdiEu P., « Le mystère du ministère », art. cit., p. 10.

51Ibid., p. 11. 